\title{
Environmental Forcing, Invasion and Control of Ecological and Epidemiological Systems
}

(Formerly titled: “The Impact of the Environment on Invasion Thresholds in Ecological and Epidemiological Systems”)

\author{
J. V. Greenman* \\ Department of Computing Science and Mathematics, \\ University of Stirling. \\ Stirling, FK9 4LA, Scotland, UK \\ tel: +44(0)1786-467460; fax: +44(0)1786-464551; \\ email: j.v.greenman@stir.ac.uk \\ and \\ R. A. Norman \\ Department of Computing Science and Mathematics, \\ University of Stirling. \\ Stirling, FK9 4LA, Scotland, UK \\ tel: +44(0)1786-467466; fax: +44(0)1786-464551; \\ email: ran@maths.stir.ac.uk
}

Keywords: environmental forcing, invasion, control, apparent competition, resonance, predation, pathogens.

Running Title: Invasion Thresholds

* Corresponding author

Submitted: 20 September 2006

Revised: 06 February 2007 


\section{Abstract.}

Destabilising a biological system through periodic or stochastic forcing can lead to significant changes in system behaviour. Forcing can bring about coexistence when previously there was exclusion, it can excite massive system response through resonance, it can offset the negative effect of apparent competition and it can change the conditions under which the system can be invaded. Our main focus is on the invasion properties of continuous time models under periodic forcing. We show that invasion is highly sensitive to the strength, period, phase, shape and configuration of the forcing components. This complexity can be of great advantage if some of the forcing components are anthropogenic in origin. They can be turned into instruments of control to achieve specific objectives in ecology and disease management, for example. Culling, vaccination and resource regulation are considered. A general analysis is presented, based on the leading Lyapunov exponent criterion for invasion. For unstructured invaders a formula for this exponent can typically be written down from the model equations. Whether forcing hinders or encourages invasion depends on two factors: The covariances between invader parameters and resident populations and the shifts in average resident population levels brought about by the forcing. The invasion dynamics of a structured invader are much more complicated but an analytic solution can be obtained in quadratic approximation for moderate forcing strength. The general theory is illustrated by a range of models drawn from ecology and epidemiology. The relationship between periodic and stochastic forcing is also considered. 


\section{Introduction.}

Most simple models of population dynamics and the spread of infectious diseases do not include any seasonal forcing. However, in reality, particularly in wildlife systems, any of the parameters might be subject to changes over a course of a year, or even longer. For example, birth rates are likely to be higher in spring and lower in winter, whereas death rates might be higher in winter and lower in summer. Disease transmission rates are also likely to vary over a season, for a number of reasons. A species may be more gregarious in spring and summer during the mating season, individuals may be less fit in the winter and therefore more susceptible to disease, or the disease might benefit from some environmental conditions rather than others. Although seasonality is the most familiar example of periodic external forcing, affecting most regions of the world and most species, there are other examples that are specific to particular regions and particular species, for example El Nino, the North Atlantic oscillation and African rainfall patterns (Wichmann et al. 2003), all with multi-annual periods. Species with multiple breeding cycles during a year and experiencing seasonal forcing can also be considered to be subject to multiple-period forcing if we take the measure of time to be the period of the breeding cycle rather than the forcing period. There is a second type of "external” force arising, for example, when a species attempts to invade a resident community or web. Not only can this species be directly subject to natural external forces but it can also be subject to fluctuations in the resident population(s) generated either by the external forces or from within the community, i.e. endogenously. As far as the invader is concerned, the resident community is part of the external environment. There is also a third type of “external” force, the result of anthropogenic intervention to achieve objectives in 
biodiversity, disease control or economic exploitation. This type of forcing will be of particular interest to us in this study.

The core problem is understanding how biological systems respond to the imposition of external periodic forcing. After much research effort, it has now become clear that the response can be far from simple or intuitive. For example, even weak forcing can have a major impact on a system when there is resonance between the system and the external forcing. Resonance occurs when the forcing period and the natural period of the system match and gives rise to amplification of the otherwise weak population oscillations (Greenman et al. 2004, Dushoff et al. 2004, Altizer 2006). With strong enough forcing the nonlinear structure of the system can be triggered, with the generation of subharmonic dynamical states (with periods that are integer multiples of the forcing period) and multiple attractors as resonance peaks overlap (Greenman et al. 2004). The relevance of nonlinear resonance to epidemiology was demonstrated by Dietz (1982), who explained the two yearly cycle of measles as an example of the subharmonic phenomenon, and by much subsequent work in the area of childhood diseases (e.g. Grenfell et al. 1995, Earn et al. 2000) and wildlife diseases (e.g. Ireland et al. 2004, Roberts and Kao 1998).

The impact that external forcing can have on a system is further illustrated by studies of particular nonlinear phenomena. For example, apparent competition, mediated by a predator, was identified by Holt $(1977,1984)$ as a mechanism by which one prey reduces the population density of another prey, possibly to the point of extinction, even though the two prey do not compete directly but interact only via the predator. This result holds when the (unforced) system is in stable equilibrium but if the equilibrium is 
unstable, with the system executing endogenous cycles, then this negative effect may not operate (Abrams et al. (1998)). It may also not operate if external forcing is applied to the system, even if it is otherwise in stable equilibrium (Brassil 2006).

Strong forcing can also influence when a species invades a resident subsystem, by raising or lowering the invasion threshold. The position of the invasion threshold, in the absence of forcing, has been a major area of research for several decades with attention being focused particularly on epidemiology (Anderson and May 1981, 1986). When the system is unforced it has been possible, in many cases of interest, to obtain an analytic expression for the infection invasion threshold. For homogenous populations this is usually expressed in terms of the basic reproduction number $\mathrm{R}_{0}$, an index that can be generalised in terms of the second generation matrix (Diekmann et al. 1990) when the vulnerable population is heterogeneous. One recent interesting application of this generalisation has been the problem of how best to eradicate a disease shared by multiple hosts (Dobson 2004). The theory has been extended to include periodic forcing (Farkas 1994) in continuous time models in epidemiology (Heesterbeek and Roberts 1995b) and in ecology (Rinaldi et al. 1993) and developed to allow a biological interpretation of the mathematical entities involved and transparency in the calculations that need to be carried out for particular applications (Heesterbeek and Roberts 1995a, 1995b; Williams and Dye 1997). Invasion in discrete time systems has also been considered, both in general and in detail for a range of models when the forcing is stochastic (Chesson 1984, 1989). Whether the model is discrete or continuous or whether the forcing is periodic or stochastic, it is now clear that external forcing can have a major impact on the ability of a species or pathogen to establish itself within a resident community. 
In this paper we seek greater understanding of how external forcing affects the invasion properties of a biological system, especially when there is more than one way in which the environment is influencing the system. It is the interaction between these different influences that brings about diversity and complexity in the system response. We will mostly restrict attention to continuous time systems under periodic external forcing, carrying out the analysis in the rare invader approximation where it is assumed that the number of invaders entering the resident community is initially small. The method of solution of the consequent dynamical equations depends on whether the resident and invading populations are structured or unstructured. By unstructured we mean that the population in question can (to a reasonable approximation) be described by a single variable and its dynamics by a single equation. A structured population is one modelled by at least two variables and two equations.

In section 2 we look at models with unstructured resident and invader and determine whether and, if so, how the invasion threshold is affected by forcing. The discussion is based on simple models from ecology and epidemiology with the general theory for (unstructured) invaders given in Appendices A, B. In section 3 we reorientate our approach by imagining that some forcing components are anthropogenic in origin rather than environmental, allowing us to use the results of section 2 to tackle the problem of how to control a system through periodic forcing, in particular how to eliminate a pathogen or unwanted predator. Vaccination, culling, resource management are strategies that will be considered. In section 4 we tackle the mathematically more difficult problem where the invader is structured and the dynamics multidimensional. Models of infection with latent period and with multiple hosts are taken as examples. In 
section 5 we study the effect of forcing on apparent competition in a prey-prey-predator model and the effect on invasion of resonance in a resident prey-predator subsystem of this model. Finally, in section 6 we argue that knowledge of the system response to periodic forcing across the full frequency range can give insight into the response of the system under stochastic forcing (Greenman and Benton 2005a, 2005b).

\section{The effect of forcing on the invasion threshold.}

\subsection{Fundamentals.}

We model periodic external forcing by simple sinusoidal variation acting through specific parameters. Precisely, if a parameter is affected by forcing then this parameter is multiplied by the factor:

$$
\mathrm{E}(\mathrm{t})=1+\delta \cos ((2 \pi \mathrm{t} / \mathrm{p})+\phi)
$$

where (as always) $\mathrm{t}$ denotes time while the amplitude $\delta$ measures the strength of forcing, $\mathrm{p}$ its period and $\phi$ its phase. Parameter $\delta$ is limited to the range $(0,1)$. Clearly the average of $\mathrm{E}(\mathrm{t})$ is 1 and the average of the forced parameter is its original value, when the average is taken over a cycle. The phase $\phi$ of the forcing is often of great significance. If two forced parameters have phases $\phi_{1}, \phi_{2}$ then they will be "in phase" if $\phi_{1}=\phi_{2}$ and (exactly) "out of phase" if $\phi_{2}=\phi_{1}+\pi$. In the latter case there is a half cycle lag between forcing maxima.

We study periodic forcing over a wide range of periods since, as explained previously, it is not only seasonality that is of interest. A biological system can experience multi-annual fluctuations generated environmentally while an invader can, in addition, be affected by high period cycles generated or mediated by the resident 
subsystem. The connection with stochastic forcing also requires understanding the system response across the full frequency (period) spectrum.

In the rare invader approximation it is supposed that the number of individuals attempting invasion is initially so small that they have no significant impact on the resident community or web, the dynamics of which can, as a result, be considered independently of the invader. Further, the invader equations can be linearised with respect to the invader populations but, with forcing, the parameters will be time dependent (i.e. the invader subsystem will be non-autonomous). If the invader is unstructured (i.e. its equation is 1-dimensional) then the invader equation can be solved and, for Lotka-Volterra type models at least, the condition for invasion can be expressed in terms of averages of and covariances between time varying parameters and resident population(s) (Appendix B). If the invader is structured then, in general, numerical methods have to be used to solve the coupled invader equations in order to determine whether invasion will take place (Hale 1969). However, an approximate but analytic solution can be obtained for moderate strength forcing (Appendix D).

We start off the analysis with the simplest case, restricting attention to systems where both invader and resident are unstructured, using formulae (B3), (B4) in the Appendix as the basis of the analysis.

\subsection{Forcing in ecological systems.}

Consider a predator attempting to invade a prey population in the absence of forcing on either population. Invasion will take if the predator intrinsic growth rate $\xi_{0}$ is positive. In the simplest case $\xi_{0}$ takes the form: 


$$
\xi_{0}=\mathrm{c}_{0} \mathrm{~N}_{0}-\mathrm{m}_{0}
$$

where $\mathrm{c}_{0}$ is the predation parameter (measuring predation success), $\mathrm{m}_{0}$ predator mortality and $\mathrm{N}_{0}$ the resident (prey) population in equilibrium at its carrying capacity level in rare invader approximation (Holt et al. 2003). The term $\mathrm{c}_{0} \mathrm{~N}_{0}$ in (2) is, in effect, the predator per capita birth rate arising from predation. (Note that the invasion condition $\xi_{0}>0$ translates into the familiar $\mathrm{R}_{0}$ condition: $\mathrm{R}_{0}=\mathrm{c}_{0} \mathrm{~N}_{0} / \mathrm{m}_{0}>1$.)

If the system is periodically forced then the condition for invasion becomes $\xi_{\text {ave }}>$ 0 where:

$$
\xi_{\mathrm{ave}}=(\mathrm{c} \mathrm{N})_{\mathrm{ave}}-\mathrm{m}_{\mathrm{ave}}
$$

Here, the average ("ave”) is to be taken over a population cycle, $\mathrm{N}$ is the (possibly varying) prey population, $\mathrm{c}$ is the (possibly varying) predation parameter with average $\mathrm{c}_{0}$ and $\mathrm{m}_{\mathrm{ave}}=\mathrm{m}_{0}$. (See Appendix B for justification of (3).) In general, if both $\mathrm{c}$ and $\mathrm{N}$ vary in time then growth rates $\xi_{0}$ and $\xi_{\text {ave }}$ will differ in value and, as a consequence, invasion will be affected by the forcing. (The situation with both c, $\mathrm{N}$ varying is of biological relevance. Seasonally varying predation is exemplified (in the extreme) by winter hibernation while seasonally driven prey oscillations can occur in a variety of ways as we will see later.)

To illustrate how external forcing can bring about a change in invasion behaviour consider the simplest nontrivial case where the prey population, $\mathrm{N}$, oscillates sinusoidally about its unforced level with the same period and amplitude, $\delta$, as the external force on predation (c) and in phase with that force. (See (1) and Appendix C for definition of the forcing parameters.) A straightforward evaluation of (3), given in Appendix C, shows that: 


$$
\xi_{\text {ave }}=\mathrm{c}_{0} \mathrm{~N}_{0}\left(1+\delta^{2} / 2\right)-\mathrm{m}_{0}
$$

So forcing aids invasion through an enhanced average predator per capita birth rate (cf (2)). How this comes about can be seen from Fig 1a which shows graphically how birth rate varies over a cycle. On the up part of the cycle there is a double (nonlinear) effect with higher prey numbers together with higher predation success raising the predator "birth rate" at its peak to four times its value with no forcing, if for definiteness we take $\delta$ $=1$. On the down part of the cycle lower success and lower prey numbers reduces the "birth rate" to zero at its lowest point (when $\delta=1$ ) but, overall, there is a net increase in births in each cycle (compared to the no forcing case). This behaviour is reminiscent of the "storage" effect (Chesson 1984). (See Appendix C for the algebra behind Fig 1a.)

If forcing on c and $\mathrm{N}$ is out of phase then $\xi_{\text {ave }}$ becomes:

$$
\xi_{\text {ave }}=c_{0} N_{0}\left(1-\delta^{2} / 2\right)-m_{0}
$$

Forcing now impedes invasion by lowering the predator average per capita birth rate. In fact this birth rate is reduced at (almost) all points in the cycle (Fig 1b). The mismatch between lower (higher) predation success but higher (lower) prey numbers always results in a loss of predation "births" compared to the unforced case. (Again see Appendix C for the algebra.)

In the calculations leading to $(4 \mathrm{a}, 4 \mathrm{~b})$ we looked at cases where forcing did not change the average of the varying prey population but this is not likely to be the case if forcing is strong (although there are exceptions to be discussed later). To separate out this effect we expand the master formula (3) to read as:

$$
\xi_{\text {ave }}=\xi_{0}+\mathrm{c}_{0}\left(\mathrm{~N}_{1}-\mathrm{N}_{0}\right)+(\mathrm{c}, \mathrm{N})_{\mathrm{cov}}
$$


where $\xi_{0}$ is the unforced growth rate given in (2), $\mathrm{N}_{1}\left(\mathrm{~N}_{0}\right)$ is the average prey population with (without) forcing and $(\mathrm{c}, \mathrm{N})_{\mathrm{cov}}$ is the covariance between variations in the predation parameter and prey population (Chesson 1989). (See Appendix B for details).

The equations for the invasion threshold (separating the regions in parameter space where invasion can and cannot take place) is given by $\xi_{0}=0$ in the absence of forcing and by $\xi_{\text {ave }}=0$ with forcing. If $\xi_{\text {ave }}>0$ when $\xi_{0}=0$ there is no invasion unless the system is forced. So forcing makes invasion easier and we say that the threshold has been lowered. If, on the other hand, $\xi_{\text {ave }}<0$ when $\xi_{0}=0$ invasion is not possible with forcing but without forcing the system is on the "threshold" of being invaded. Invasion has been made more difficult by the forcing, i.e. the threshold has been raised.

Whether the threshold is raised or lowered depends on the sign of the sum of the two terms giving the change in growth rate $\left(\xi_{\text {ave }}-\xi_{0}\right)$ in (5). Typically the first term is negative $\left(\mathrm{N}_{1}<\mathrm{N}_{0}\right)$ due to the "spiked" nature of the time series generated by strong forcing, with the prey spending only short periods at high levels, spending most of the time at low levels. The covariance in the last term of (5) is related to the phase between the forcing components on $\mathrm{c}$ and $\mathrm{N}$. If the forcing is in phase (out of phase) the covariance is likely to be positive (negative) as (4a), (4b) show.

\subsection{Forcing configurations.}

To show how different forcing configurations on a system lead to different invasion outcomes, let us consider, for definiteness, the predator prey Model 1 defined in Table 1. In rare invader approximation the prey is logistic, satisfying the equation: 


$$
\mathrm{dN} / \mathrm{dt}=\mathrm{r} \mathrm{N}(1-\mathrm{N} / \mathrm{K})
$$

where $\mathrm{N}$ denotes the prey population, $\mathrm{r}$ the prey intrinsic growth rate and $\mathrm{K}$ the prey carrying capacity. Suppose that only the growth rate $\mathrm{r}$ is forced with $\mathrm{K}$ fixed. (This would be the case if $\mathrm{K}$ models, say, physical limitations on a prey habitat (e.g. cliff or island).) In the long run there will be no variability in the prey population since any oscillation that occurs will be damped by the density dependent factor $(1-\mathrm{N} / \mathrm{K})$ in $(6)$. So by (5), with $\mathrm{N}$ constant at its carrying capacity level (i.e. $\mathrm{N}_{1}=\mathrm{N}_{0}$ ), there will be no shift in the threshold whether or not the predation parameter c is forced.

Suppose, instead, that both $\mathrm{r}$ and $\mathrm{K}$ are forced in such a way that the ratio $\mathrm{s}=$ $\mathrm{r} / \mathrm{K}$ is, to a good approximation, constant in time (Bowers et al. 2003, Rinaldi et al. 1993). (This synchrony might be achieved, for example, if forcing is seasonal and $\mathrm{K}$ is a proxy for food supply.) Then one can show that $\mathrm{N}_{1}=\mathrm{N}_{0}$ (i.e. the average prey population does not change under forcing) and so, by (5), it is the sign of the covariance between $\mathrm{c}$ and $\mathrm{N}$ that determines the direction of the threshold shift (Table 2, columns A, B).

If, thirdly, the prey is forced only through $\mathrm{K}$ then the average prey population is depressed (i.e. $\mathrm{N}_{1}<\mathrm{N}_{0}$ ) and so the last two terms in (5) both contribute. As a consequence the threshold is raised (i.e. invasion is made more difficult) if c and $\mathrm{K}$ are out of phase (Table 2, column D) since the last two terms in (5) are both negative. If they are in phase then there can be a raising or a lowering of the threshold, depending on the magnitudes of the last two terms in (5), now having opposite signs. Table 2, column C shows that their sum depends on the period and that increasing the period can switch the 
system from a raised to a lowered threshold with the final (positive) term in (5) becoming dominant.

Finally, we note that there can be a shift in the threshold even if c is not forced provided the prey is forced only through $\mathrm{K}$. Then the second term in (5) contributes but not the third.

\subsection{Forcing in epidemiological systems.}

The results obtained for the predator prey Model 1 (Table 1) apply equally to the SIRS compartment model of microparasite infection defined by Model 2 in Table 1 . This model separates the population into susceptibles, S, infectious, I, and recovereds, R, with the possibility of loss of immunity and reversion to the susceptible state. The total population is denoted by $\mathrm{H}$ where $\mathrm{H}=\mathrm{S}+\mathrm{I}+\mathrm{R}$. For pathogen invasion the susceptibles form the resident population and the infectious population the invader. In the rare invader approximation the susceptible population, like the prey population in Model 1, satisfies the logistic equation. The equation for the infectious population is identical to the predator equation in Model 1 when $\mathrm{c}$ is replaced by the transmission rate $\beta$ and mortality $m$ by the loss rate, $d$, from the infectious state. (Note that the equation for the invader does not depend on the recovered population $\mathrm{R}$ and so the equation for $\mathrm{R}$ can be ignored as far as invasion is concerned.) Because of the identical structure of Models 1, 2 in rare invader approximation the average invader growth rate for Model 2 can be written down immediately from (3), (5) as:

$$
\xi_{\mathrm{ave}}=(\beta S)_{\mathrm{ave}}-\mathrm{d}_{\mathrm{ave}} ; \quad \xi_{\mathrm{ave}}=\xi_{0}+\beta_{0}\left(\mathrm{~S}_{1}-\mathrm{S}_{0}\right)+(\beta, \mathrm{S})_{\mathrm{cov}}
$$

with obvious notation. 
From the predator prey results we infer that there will be no change in the position of the infection invasion threshold (relative to the position where the varying parameters in the model are replaced by their average values) when $K$ is fixed nor when both $r / K$ and $\beta$ are fixed. Otherwise the infection invasion threshold is raised or lowered depending on forcing strengths, phases and configuration. The results shown in Table 3 (columns A, B, C), for example, are in line with those of Table 2 (columns A, B, D) for the predator prey model.

\subsection{Extensions.}

Throughout the discussion so far we have assumed that the interaction between invader and resident has been mass action. (Note that Begon et al. (2002) use the term "density dependent” and de Jong et al. (1995) “pseudo mass action” for this type of interaction. However we will continue to use the term "mass action" for its simplicity and to avoid other conflicts in the use of terminology.) If the interaction is not mass action how is invasion affected? Suppose the interaction in the predator prey Model 1 is of the form $\mathrm{f}(\mathrm{N}) \mathrm{P}$ where $\mathrm{f}$ is a positive decelerating function with positive gradient (e.g. Holling type II), modelling consumption per predator. Formula (5) still holds provided $\mathrm{N}$ is replaced by $\mathrm{Z}=\mathrm{f}(\mathrm{N})$. The analysis of the amended formula proceeds as before. As with mass action, there is no shift if $\mathrm{K}$ is fixed but unlike mass action there is a shift if $\mathrm{r}$ is varied with both $\mathrm{r} / \mathrm{K}$ and c fixed. This is because the amended second term in (5) is no longer zero, becoming negative because of the nonlinearity of the consumption function f (Ruel and Ayres 1999). So, certainly in this case, saturation in predator consumption makes invasion more difficult. 
The form of the interaction also matters in the SIRS model. There are strong arguments that, at least in some disease systems, the infection transmission term is not mass action (as in Model 2) but is frequency dependent, i.e. of the form $\beta \mathrm{f}(\mathrm{S}, \mathrm{H}) \mathrm{I}$ where $f(S, H)=S / H$ (Hethcote et al. 2005). Such a remodelling would have a major impact on infection invasion since, in the rare invader approximation, $\mathrm{f}(\mathrm{S}, \mathrm{H})=1$ and so all variation is averaged out. However the frequency dependent form of the transmission term is not biologically valid at low susceptible population levels, the situation for much of the cycle under strong forcing. There is therefore not likely to be much of a difference when saturation is present in the interaction. This can be checked out by supposing that the transition between high and low population levels can be captured by the interaction term: $\beta g(H) S I / H$ where $g(H)=H /(1+h H)$. (See Dietz (1982).) The results, with $h=$ 0.1 and with the scenario defined in column B in Table 3, are listed in column D of that table.

One should also note that, certainly for the SIRS model, it matters how self regulation through density dependence is introduced into the model. If density dependence works through mortality rather than reproduction, i.e. $b=b_{0}+b_{1} H$, then there will be an extra term appearing in (5) if there is variation in both population $\mathrm{S}(\sim$ H) and parameter $b_{1}$. We can absorb this extra contribution in the transmission term, thereby amending the transmission rate $\beta$. The situation is different for the predator prey Model 1. If there is density dependence in predator mortality, i.e. $\mathrm{m}=\mathrm{m}_{0}+\mathrm{m}_{1} \mathrm{P}$ (where $\mathrm{P}$ is the predator population) there is no consequent additional shift in threshold since any variation in $\mathrm{m}_{0}$ is averaged out and the $\mathrm{m}_{1}$ term can be ignored in the rare invader approximation. 


\section{Control of environmentally forced systems.}

\subsection{Ecological control.}

We can use the results we obtained (Table 2) for the predator prey Model 1 (Table 1) to address the problem of whether we can control a predator prey system when some components of the forcing are anthropogenic in origin rather than environmental. For example, suppose that the prey carrying capacity is periodically forced by human intervention, through, for example, varying resource availability in a wildlife or laboratory setting while predation continues to be environmentally forced. In these circumstances the analysis suggests that it may be possible to control or eliminate the predator by raising its invasion threshold (Table 2, column D) if, for whatever reason, it is not possible to directly control the predator. To achieve this, there has to be a large enough phase difference between control and environmental forcing.

Alternatively it may be possible to control a predator population by periodic culling of the prey. This possibility relates to the first calculation we carried out where parameters $\mathrm{r}$ and $\mathrm{c}$ are varied while $\mathrm{r} / \mathrm{K}$ is kept fixed (Table 2, column B). The threshold shift is quite small for low periods but increases with period, in contrast to the response to resource management where there is a decrease with period (Table 2, column D). The shift at low periods can be increased by modelling culling more accurately by varying prey mortality rather than net growth. This will have most effect when per capita mortality and birth rates are comparable so that the intrinsic growth rate, $r$, is negative over a substantial part of the cycle. Control can be further enhanced if the culling is 
“pulsed”, i.e. it happens after every so many years. This strategy can be modelled by using the exponential factor:

$$
\mathrm{C} \exp \left(\delta_{2} \cos ((2 \pi \mathrm{t} / \mathrm{p})+\phi)\right)
$$

rather than the sinusoidal form (1) (Anderies and Beisner 2000). The scaling factor $C$ in (8) is chosen to normalise (8) to unity over a cycle. The greater the parameter $\delta_{2}$ the closer this forcing factor is to a pulse every p "years". The effect of pulsing can be seen in Table 2, columns E, F when the second parameter set in Table 1 (Model 1) is used.

\subsection{Epidemiological control.}

There are various ways of tackling the problem of eliminating a pathogen, vaccination being the one most widely used when feasible. For childhood diseases this is typically carried out at or within a few years of birth. However the proportion of children that have to be vaccinated can be very high, as in the case of measles. An alternative strategy, instead of or as well as vaccination "at" birth, is to vaccinate a proportion of the susceptible population periodically (Shulgin et al. 1998, Moneim and Greenhalgh 2005a). This can be considered to be an example of external periodic forcing with vaccination moving susceptibles, $\mathrm{S}$, to a redefined "immune" class, $\mathrm{R}$, consisting of individuals that have been vaccinated or have recovered from the disease and have (at least) temporary immunity. The resident is now structured, consisting of both S and R populations, because of the link between these classes through vaccination. An "extreme" form of periodic vaccination is to vaccinate a given proportion of the susceptible population on the same day of the year every so many years. We can approximate this "pulse" vaccination strategy by the function: $v(t)=C \exp \left(\delta_{2} \cos (2 \pi t /(m p)+\phi)\right)$ which is scaled, 
through factor C, to achieve a given overall vaccination target. The vaccination period, $\mathrm{mp}$, is an integer multiple, $\mathrm{m}$, of the environmental forcing period $\mathrm{p}$. The larger $\delta_{2}$ the sharper the pulse.

To determine the effect of periodic forcing through vaccination, in addition to direct environmental forcing on the transmission rate $\beta$, we use formula (7) where the susceptible population $\mathrm{S}$ is found by numerically solving the two coupled dynamical equations for the resident subsystem (S, R). For the parameter values specified in Table 1 (Model 2) we obtain the results listed in Table 3 (columns E, F) giving the critical (threshold) values of the (average) transmission rate $\beta_{\text {ave }}$ against forcing period. Table 3 shows the efficacy of periodic vaccination (column E) in raising the invasion threshold but this effect falls off when the frequency of the vaccination is less (column F) or when the environmental forcing period is higher (columns E, F).

Two alternatives to vaccination in the control if not prevention of a disease have been suggested in the discussion of the predator-prey model: Culling (in non-human populations) (Roberts and Kao 1998) and resource accessibility management, but the timing of any intervention has to be well-judged for the threshold to be raised. In practice a hybrid strategy is likely to be adopted with periodic control coupled with additional measures to reduce parameter and population averages. Pulsed vaccination together with vaccination at birth is an example of how this can be carried out to advantage (Shulgin et al. 1998).

\section{Structured invaders.}

\subsection{Inclusion of a latent state in the SIRS model.}


Mathematically, the analysis takes on a different form if a latent (exposed) stage, E, in which the population is infected but not (yet) infectious, is added to Model 2, yielding the SEIRS Model 3 of Table 1. For this model the invader is structured, now consisting of populations E, I satisfying the differential equations:

$$
\begin{aligned}
& \mathrm{dE} / \mathrm{dt}=\beta \mathrm{IS}-(\gamma+\mathrm{b}) \mathrm{E} \\
& \mathrm{dI} / \mathrm{dt}=\gamma \mathrm{E}-(\mu+\mathrm{b}+\alpha) \mathrm{I}
\end{aligned}
$$

with the susceptible population, S, in (9a) found separately, in rare invader approximation. The two invader equations are linear in populations E, I but, under forcing, their parameters are time dependent (i.e. the equations are non-autonomous). This means that, in general, they cannot be solved explicitly in terms of time averages or covariances. In particular they cannot be solved by replacing the time varying parameters by their time averages nor can the $\mathrm{R}_{0}$ condition for invasion be obtained by replacing the terms in the unforced expression for $\mathrm{R}_{0}$ by their time averages although in some situations this might provide a good approximation (Moneim and Greenhalgh 2005b, d'Onofrio 2002). So these equations have to be solved numerically, either by simulation to find the long term behaviour or, equivalently, reduced to a discrete time problem by taking snapshots of the invader after each successive cycle. Invasion occurs if the matrix, Q, that moves the invader along its trajectory in state space between snapshots becomes unstable. This matrix, related to the Fundamental Matrix of the invader equations, is found by solving these equations over a population cycle (Farkas 1994). (See Appendix B for details.) The critical value of $\beta$, indicating the onset of invasion, occurs when the dominant eigenvalue of matrix Q, takes value 1 . 
The results obtained for the SEIRS model, using the parameter values of Table 1 (Model 3), are qualitatively similar to those for the SIRS model for the forcing configurations of Table 3 although the phase differences that maximise the threshold shift are substantially perturbed. The SEIRS model does, however, exhibit new behaviour. The threshold can be raised when there is forcing only on the transmission rate $\beta$ (with $\mathrm{S}$ not varying). This contrasts with no shift at all for the SIRS model. Increased dimensionality allows oscillations to develop within the structured invader subsystem, oscillations that affect the onset of invasion.

This result can be strengthened if we take a quadratic approximation in the forcing strength $\delta$, enabling invader equations $(9 \mathrm{a}, \mathrm{b})$ to be solved analytically (Appendix D). In this approximation one can show that the threshold is always raised.

\subsection{Two host single pathogen model under forcing.}

An important problem, arising from recent examples of the spread of disease amongst wildlife and livestock, is how best to eliminate a pathogen by controlling some but not all of the hosts sharing the pathogen. Such a situation might arise because of cost, accessibility or some other restricting circumstance (Dobson 2004). Here we look at a simplified version of this problem with just two hosts but with the added complication of external forcing. The model we use is set out as Model 4 in Table 1 and has been fully analysed, when unforced, in Greenman and Hudson (1999). In this model we suppose, for simplicity, that there is only one stage in the progression of the disease, i.e. there is no latency and no immunity. When unforced the dynamical equations for invading populations $I_{1}, I_{2}$ are given by: 


$$
\begin{aligned}
& \mathrm{dI}_{1} / \mathrm{dt}=\mathrm{K}_{1}\left(\beta_{11} \mathrm{I}_{1}+\beta_{12} \mathrm{I}_{2}\right)-\mathrm{d}_{1} \mathrm{I}_{1} \\
& \mathrm{dI}_{2} / \mathrm{dt}=\mathrm{K}_{2}\left(\beta_{21} \mathrm{I}_{1}+\beta_{22} \mathrm{I}_{2}\right)-\mathrm{d}_{2} \mathrm{I}_{2}
\end{aligned}
$$

with the susceptible populations at their carrying capacity levels, $\mathrm{K}_{\mathrm{i}}$, in the rare invader approximation. The matrix of coefficients, $M$, of $I_{1}$ and $I_{2}$ in $(10 a, b)$ is related to the second generation matrix frequently used in analysing invasion in heterogeneous populations (Diekmann et al. 1990, Dobson 2004). When unforced, invasion occurs when the leading eigenvalue of $\mathrm{M}$ has positive real part.

Equations (10a, b) still hold if there is forcing only on the intra- and inter-

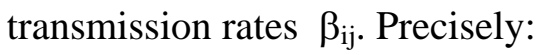

$$
\beta_{\mathrm{ij}}=\beta_{\mathrm{ij}(\text { ave) }} \mathrm{E}_{\mathrm{i}} \text { where } \mathrm{E}_{\mathrm{i}}=1+\delta_{\mathrm{i}} \cos \left((2 \pi \mathrm{t} / \mathrm{p})+\phi_{\mathrm{i}}\right), \quad(\mathrm{i}=1,2), \phi_{1}=0.0
$$

The equation system (10a, b) now has to be solved numerically, without the advantage of eigenvalue theory, since, as with the SEIRS model, the invader subsystem is nonautonomous and has dimensionality greater than one. For the parameter set in Table 1 (Model 4) we find a lowering of the threshold for all values of $\phi_{2}$ considered, especially when the forcing terms are out of phase $\left(\phi_{2}=\pi\right)$ (Table 4, columns A, B). (Again one can show that these results hold, whatever the parameter values, in quadratic approximation.) However if forcing is also applied to the growth rate of just host 1 (with the density dependent coefficient r/K kept fixed), i.e.

$$
r_{1}=r_{1(\text { ave })} E_{3} \text { where } E_{3}=1+\delta_{3} \cos \left((2 \pi t / p)+\phi_{3}\right)
$$

then the lowering of the threshold can be reversed and the threshold raised if the forcing on $r_{1}$ is out of phase with the forcing on the transmission rates (Table 4, column D), an effect that increases with forcing period. (With the forcing in phase the threshold remains lowered (Table 4, column C).) The impact can be increased at low forcing 
periods if it is the carrying capacity, $\mathrm{K}_{1}$, that is controlled (Table 4, column E). These results suggest that single host periodic culling and resource manipulation are possible ways of managing a disease shared by two hosts, made worse by the presence of environmental forcing on disease transmission.

We noted that for Models 1, 2 with just one prey and predator and just one host and pathogen (resp.) there was an identity of structure that led to identical invasion behaviour under forcing. However, models describing two hosts with shared pathogen (Model 4, Table 1) and two prey with shared predator (Model 5, Table 1) do not have a common structure even in rare invader approximation. As we have seen, the pathogen invader in Model 4 is structured while in Model 5 the predator invader is unstructured. The contributions of the two prey to the predator "birth rate" in Model 5 are additive. There is, as a result, no shift in the predator invasion threshold if forcing is only on the predation parameters, $\mathrm{c}_{1}, \mathrm{c}_{2}$, in contrast to Model 4 where there is a shift if forcing is only on the transmission rates.

\section{External forcing and apparent competition.}

\subsection{A predator-prey-prey model.}

To study apparent competition we continue the analysis of Model 5 in which two (noncompetitive) prey share a single predator. We note first that there are invasion thresholds for each of the component populations. Their typical positions and the nature of the dynamics in the regions defined by them are shown in Fig 2 when there is no forcing. Fig 2 shows the cross-section of parameter space defined by the predation parameters $c_{1}$, $\mathrm{C}_{2}$ (with the other parameters fixed). 
In region $\mathrm{R}$ of Fig 2 the predator is unable to invade; in Region $\mathrm{S}$ the predator coexists with both prey; in regions $\mathrm{T}$ and $\mathrm{U}$ only one prey can persist in the long run, prey 1 in region $\mathrm{T}$ and prey 2 in region $\mathrm{U}$. Boundary $\mathrm{b}_{1} \mathrm{~b}_{1}$ (between regions $\mathrm{R}, \mathrm{S}$ ) is the predator invasion threshold, boundary $b_{2} b_{3} b_{4}$ the invasion threshold for prey 2 while boundary $b_{5} b_{5}$ is the threshold for prey 1 . Regions $T, U$ illustrate the "extreme" form of apparent competition, mediated by the predator: One prey can exclude the other prey even though there is no direct competition between them (Holt 1977). More generally, invasion of one prey will have a negative impact on the other prey, through apparent competition, by lowering its population level without necessarily forcing it to extinction (region S) (Holt 1984).

To study apparent competition under external forcing we first focus on section $\mathrm{b}_{3} \mathrm{~b}_{4}$ of the prey 2 invasion threshold (Fig 2). The interacting resident populations, prey 1 and the predator, can generate quite complex dynamics but the single invader equation for prey 2 (in rare invader approximation) can be solved to yield the prey 2 average growth rate:

$$
\xi_{\text {ave }}=\xi_{0}-\mathrm{c}_{2 \mathrm{ave}}\left(\mathrm{P}_{1}-\mathrm{P}_{0}\right)-\left(\mathrm{c}_{2}, \mathrm{P}\right)_{\mathrm{cov}}
$$

$\mathrm{P}$ is the predator population, $\mathrm{P}_{1}$ its average under forcing and $\mathrm{P}_{0}$ without forcing. If the predation parameter $c_{2}$ is unforced but there is forcing on the prey intrinsic growth rates, $\mathrm{r}_{1}, \mathrm{r}_{2}$, then the threshold is lowered since, in (11), $\mathrm{P}_{1}<\mathrm{P}_{0}$ and the covariance is zero. (Note that the resident is no longer logistic.) This means that the forcing is offsetting apparent competition by allowing prey coexistence when, without forcing, this would not be possible. In geometric terms, external forcing is expanding the coexistence region S by pushing out the $b_{3} b_{4}$ boundary in Fig 2 into region $T$. As a numerical example we list 
in Table 5 (column A) the (critical) threshold values of $\mathrm{c}_{1}$ for the parameter values listed in Table 1 for Model 5 (when forcing is only on the prey intrinsic growth rates).

If forcing is applied to the predation parameters as well as the prey growth rates then the third term in (11) contributes to the threshold shift. Its sign is very sensitive to the phase between the two forcing terms. For example, for the parameter set of Table 1 (Model 5) and with the forcing on the growth rates lagging behind the forcing on predation by a quarter cycle there is reinforcement with the threshold further lowered (Table 5, column C). If, instead, the forcing terms are in phase then there is little or no shift in the threshold position (a result not shown in Table 5).

\subsection{The implications of nonlinear resonance.}

The lowering of the threshold shown in Table 5 (columns A, C) depends in an unsmooth manner on the forcing period. This is a consequence of resonance in the resident subsystem. To understand the connection, we first review the essential features of nonlinear resonance.

Resonance is a phenomenon well known to occur in linear systems when the period, $\mathrm{p}$, of an external force is close to the natural period, $\mathrm{p}_{0}$, of the dominant decay mode of the stable system to which it is applied (Greenman et al. 2004). Its presence is revealed in the response curve of the system, in which the maximum reached by one of the populations defining the system is plotted against forcing period. There will be a peak in this curve about the natural period, its prominence increasing the slower the decay mode to which it relates. So resonance implies amplification of the population oscillations when the forcing and natural periods match. 
If the system is nonlinear but the forcing is weak then the system can be linearised about its equilibrium and the peak is then symmetric about the natural period. However, if the forcing is increased then nonlinearities in the system lead to distortion of the peak which progressively leans to the right (to higher forcing periods) as its height increases (Fig 3a), eventually overlapping with itself to create multiple attractors (Fig 3b). At the same time a second peak emerges from the point at $\mathrm{p}=\mathrm{p}_{0} / 2$ (on the period axis of the response curve) and grows with the strength of the forcing, also suffering asymmetric distortion in the process (Fig 3b). This second peak corresponds to oscillations that have period twice that of the external forcing, i.e. they define a "subharmonic" of the forcing.

The peaks "rooted" at $\mathrm{p}=\mathrm{p}_{0}, \mathrm{p}_{0} / 2$ are just two of a hierarchical family of "peaks" that emerge as the forcing strength is increased. Details of their shape and structure can be found in Greenman et al. (2004). Of particular interest is the fact that, through distortion, different peaks can overlap, indicating the presence of another level of multiple attractors, with both large and small amplitude oscillations possible. Switching between these different modes can happen through external shocks.

We now have an explanation for the results of Table 5. The prey1-predator resident subsystem in Model 5 is experiencing strong resonance under the impact of external forcing. The prey 1 response curve is shown in Fig 4 based on a natural period of $\mathrm{p}_{0} \sim 12$ with forcing on just the prey growth rates. We note the presence of a subharmonic with period twice the forcing period, over the range $5<\mathrm{p}<11$. The distortion of the peak of this subharmonic is so great that overlapping occurs with the creation of two possible dynamic states for forcing period $\mathrm{p} \sim 11$. The shift in the prey 2 invasion threshold value of $c_{1}$ under forcing is superimposed on Fig 4 and shows strong 
negative correlation with the prey 1 response curve. The larger the amplitude of the resident oscillations the more the threshold is lowered.

We have observed in previous models that changing the phase between forcing terms can significantly alter the dynamics of invasion. Model 5 shows that this can also be the case if we vary the strength of forcing. For example if, when the external forces on predation parameters and growth rates are in phase, we reduce the strength of the growth rate forcing then the overall very weak threshold effect (previously mentioned) is transformed into a strong uplift in the threshold. Further reduction in strength however leads to a switch to a lowered threshold due to the double period (2p) subharmonic being triggered in the resident subsystem. In between, there is an overlap of resonance peaks with the possibility of the system switching between lowered and raised threshold states. This happens, for example, for the model parameter set listed for Model 5 in Table 1 and forcing parameters $\left(\delta_{1}, \delta_{2}, \mathrm{p}\right)=(0.35,0.95,10)$ where $\delta_{1}, \delta_{2}$ are the forcing strengths on growth rates and predation parameters (resp.).

We have been considering boundary $b_{3} b_{4}$ in Fig 2 . On the remainder of the prey 2 invasion boundary, $b_{2} b_{3}$, resonance, certainly for just growth rate forcing, is weak and there is only a small shift in the threshold and very little period dependency. However a remarkable phenomenon happens if we apply forcing on both the carrying capacities and the predation parameters but not on the growth rates. Then there is a strong response in terms of the amplitude of the resident oscillations but a weak response in terms of resonant "peak" structure. For low periods $(p<15)$ this leads to the gap between $b_{2} b_{3}$ and $b_{3} b_{4}$ disappearing (with zero now the critical value of $c_{1}$ ), i.e. there is coexistence throughout much of the prey 2 exclusion region T in Fig 2 . 


\section{Stochastic forcing.}

We have seen that periodic forcing of ecological and epidemiological systems can have a substantial impact on the system, not least in the repositioning of invasion thresholds within parameter space. The question naturally arises as to whether stochastic forcing (i.e. external noise) can affect the behaviour of a system in similar ways. For simplicity we discuss only white noise, i.e. noise which has no correlation over time and, as a result, has a power spectrum spread evenly across the allowed frequency range (Caswell 2001, Hamilton 1994). (When there is correlation over time the spectrum has structure and the noise is said to be coloured (Kaitala et al. 1997). For example, correlation occurs when a dry summer is more likely to be followed by another dry rather than wet summer. The noise is then said to have a reddish hue.) The homogeneous spectrum for white noise suggests that the response of the system to white noise is an "average" of its responses to periodic forcing across the full frequency range. We test this hypothesis on the predatorprey Model 1.

To correctly handle white noise in continuous time models requires a high degree of technical skill (Arnold 1999). To side-step the issues involved we approximate white noise by assuming constancy over each successive time interval of length $h$ where $h$ is small and determined by the short term correlations implicit in the environmental fluctuations. Precisely, we take the environmental forcing factor to be:

$$
\mathrm{E}(\mathrm{t})=1+\delta \phi(\mathrm{t})
$$

where $\phi(t)$ is drawn from a random variable $\phi$ with homogeneous distribution in the range $(-1,1)$ at the beginning of each time interval. For stochastic forcing and for the 
distributions we are using, the invasion exponent, $\xi_{\text {ave }}$, defined by (B1) in the Appendix holds for a realisation of the random variable defining the white noise, with the average taken over a long time (i.e. as $\mathrm{t}$ tends to infinity) rather than over a periodic cycle (Caswell 2001, Ferriere and Gatto 1995). As a consequence, the arguments that led to no shift in the invasion threshold for Model 1 lead to the same conclusion for white noise. However a shift occurs for the forcing configurations of columns A to D of Table 2 with stochastic as well as periodic forcing. The results for these scenarios when the forcing terms have correlation +1 (columns A, C) or correlation -1 (columns B, D) are given in the last row of Table 2. They are consistent with the hypothesis that white noise averages the periodic response (taking into account the lower variance for forcing (12)). In particular the critical threshold value in column $\mathrm{C}$ is the average of a strong uplift in threshold for low periods and a modest lowering of threshold for all other periods.

The significance of the difference in correlation between the different pathways by which noise affects a system can be understood more clearly when there are two species involved. One might expect the forcing on these species to be highly correlated but there are situations when this may not be the case since the species may have different responses to the forcing, even if the forcing is affecting the same trait in the two species. For example, if the forcing is mediated through temperature one species might respond to higher temperature with higher reproduction but the other species might have the opposite reaction, i.e. the responses are then negatively correlated.

One way to model the difference between forcing and the biological response to that forcing is to introduce pre-filters (Laakso et al. 2004)) with lags if the filtering process introduces significant delays in the impact of the forcing. As an approximation 
one could include such a lag as a phase in the forcing term (1) for periodic forcing. For white noise such a lag is likely to lead to low or zero correlation between forcing components because of the absence of temporal correlation in white noise.

\section{Discussion.}

With a few important exceptions, external periodic forcing leads to a shift in the invasion threshold of a predator, prey, pathogen or infected species attempting to invade a resident community. The sign of the shift (i.e. whether the invasion threshold is raised or lowered relative to the unforced position) is clearly of importance when there is concern about maintaining biodiversity within an ecological system, about threats to economically valuable plant or animal species or about the spread of disease within and between human, livestock and wildlife communities. The sign of the shift is found to be very sensitive to the relative strengths and phase differences of the components of the forcing as they affect different aspects (i.e. different model parameters) of the system. For predator prey Model 1 and microparasite Model 2 we found that by changing the phase we could change the sign of the shift. For the two host SIS Model 4, forcing one host growth rate as well as the transmission rates can reverse the sign of the threshold shift if the added forcing is out of phase. For the predator two prey Model 5, the change in sign can be brought about by reducing the forcing strength on the prey growth rate.

This ability to change the sign of the shift in threshold by changing the forcing parameters has important consequences for the control of ecological and epidemiological systems. If one or more of the components of the forcing is under "management control" rather than being driven environmentally then it might very well be the case that 
sufficient controls are available to achieve management objectives, for example eradication of an infection or the survival of a species in an ecological web. To this end we used the Models 1, 2, 3 as a testbed to compare different methods of disease control suggested by different configurations of the forcing. In the more complex Model 4 we showed how infection might be eliminated by control of some but not all of the hosts while in Model 5 we showed that the negative effect of apparent competition could be reversed with external forcing. So the diversity and complexity of behaviour that arise when there are multiple channels through which forcing impacts on a biological system should be viewed positively, in providing an opportunity to be exploited in the control of such systems.

Model 5 also illustrates the complexity that can arise when the resident (predatorprey) subsystem is experiencing nonlinear resonance under external forcing. Not only is the magnitude of the threshold shift highly sensitive to the forcing period as a result but there can be multiple resident states due to overlapping of the resonance peaks. This can mean that there is more than one path along which the invader can invade. For one of these paths we found that the threshold is lowered and, for the other, raised.

The analysis has been based on Lyapunov theory (Ferriere \&Gatto 1995) with invasion taking place if the leading Lyapunov exponent is positive (Appendix B). This exponent generalises the notion of leading eigenvalue of the matrix describing invasion in the rare invader approximation to those situations where there is time dependence in the model parameters. For unstructured invaders this exponent is equal to the average over a cycle of the invader intrinsic growth rate which can usually be written down immediately from the invader equations. For structured invaders the leading exponent is the dominant 
eigenvalue of the Fundamental Matrix of the invasion subsystem evaluated over a population cycle (Farkas 1994)(Appendix B). To find this matrix means solving (numerically) the invader equations over a population cycle. However one can obtain an analytic but approximate solution when forcing is of moderate strength by taking a quadratic expansion in that forcing strength.

The conventional way to express the condition for invasion in an unforced system is in terms of index $R_{0}$ for an unstructured system and the spectral radius of the second generation matrix for structured systems. These can be derived from the exponent methodology and so can be generalised when forcing is present. The unity of our approach through the use of the Lyapunov exponent has enabled us to cross-link ideas and methods between ecology and epidemiology, particularly with respect to control and to explore the similarities and differences in behaviour resulting from differences in model structure between these two areas of application.

The richness of behaviour evident in the analysis and numerical exploration of the models of Table 1 warrants further examination. This is especially so for the structured Models 4 and 5 and the issues they address. In particular it is important to gain a greater understanding of how time dependence can change the behaviour of the linear dynamical system describing the invasion process. Also needed is a much more detailed study of the relationship between periodic and stochastic forcing. We have seen that there are interesting connections between these two types of forcing but have noted that system behaviour under stochastic forcing is particularly sensitive to how the biological response to the forcing is modelled and the nature of the forcing itself, in particular whether it has significant temporal correlation. 
In this paper we have not considered the situation where the resident community, when alone, is unstable, executing endogeous periodic cycles. External forcing on the resident community can convert these fluctuations into quasiperiodic or period-locked cycles (or possibly chaotic dynamics) (Greenman et al. 2004). Analysis of invasion in this situation is handeable within the exponent methodology but involves tackling such questions as to how forces with different periods interact. Except in the case of subharmonic generation, the analysis we have carried out here has assumed forces with a common period.

We have only considered smooth transitions across the invasion threshold, activated by a transcritical bifurcation. Invasion can also occur through a saddle-node bifurcation which leads to the sudden (discontinuous) emergence and persistence of a (possibly) sizeable population. Crossing such a threshold in the reverse direction results in the sudden extinction of a population even though its size might suggest that the risk of extinction is low. This second mechanism occurs in the simplest (Lotka-Volterra) two species direct competition model and is therefore likely to occur in more complex models, given the right set of circumstances.

\section{Acknowledgements.}

We would like to thank Mike Boots in Sheffield and Ben Mestel in Stirling for helpful discussions during the preparation of this manuscript. The algebraic and numerical calculations were carried out on an AppleMacintosh G4 computer using the software package Mathematica (Wolfram 1999). 


\section{References.}

Abrams, P. A., R. D. Holt, R. D., Roth,J. D. 1998. Apparent competition or apparent mutualism? Shared predation when populations cycle. Ecology 19, 201-212.

Altizer, S., Dobson, A., Hosseini, P., Hudson, P., Pascual, M., Rohani, P. 2006. Seasonality and the dynamics of infectious diseases. Ecology Letters 9, 467-484.

Anderies, J. M., Beisner, B. E. 2000. Fluctuating Environments and Phytoplankton Community Structure: A Stochastic Model. Am. Nat. 155, 556-559.

Anderson, R. M., May, R. M. 1981. The population dynamics of microparasites and their invertebrate hosts. Phil. Trans. R. Soc. Lond. B291, 451-524.

Anderson, R. M., May, R. M. 1986. The invasion, persistence and spread of infectious diseases within animal and plant communities. Phil. Trans. R. Soc. Lond. B314, 533-568.

Arnold, L. 1999. Random Dynamical Systems. Springer, New York.

Begon, M., Bennett, M., Bowers, R. G., French, N. P., Hazel, S. M., Turner, J. 2002. A clarification of the transmission terms in host-microparasite models: numbers, densities and areas. Epidemiol. Infect. 129, 147-153.

Bowers, R. G., White, A., Boots, M., Geritz, S. A. H., Kisdi, E. 2003. Evolutionary branching/speciation: contrasting results with explicit or emergent carrying capacities. Evolutionary Ecology Research 5, 883-891.

Brassil, C. E. 2006. Can Environmental Variation Generate Positive Indirect Effects in a Model of Shared Predation? Am. Nat. 167, 43-54.

Caswell, H. 2001. Matrix Population Models. Sinauer Associates, Inc., Sunderland, Massachusetts.

Chesson, P. 1984. The storage effect in stochastic population models. Lecture Notes in Biomathematics 54, 76-89. Springer, New York.

Chesson, P. 1989. A General Model of the Role of Environmental Variability in Communities of Competing Species. Lectures on Mathematics in the Life Sciences 20, 97-123. American Mathematical Society.

de Jong, M. C. M., Diekmann, O., Heesterbeek, J. A. P. 1995. How does transmission of infection depend on population size? In: Epidemic models: their structure and 
relation to data. Mollison, D. ed. Cambridge: Cambridge University Press. 1995, 89-94.

Diekmann, O., Heesterbeck, J. A. P., Metz, J. A. J. 1990. On the definition and computation of the basic reproduction ratio $\mathrm{R}_{0}$ in models for infectious diseases in heterogenous populations. J. Math. Biol. 28, 365-382.

Dietz, K. 1982. Overall population patterns in the transmission cycle of infectious disease agents. In: Population Biology of Infectious Diseases, Anderson, R. M., May, R. M. (eds.), Springer-Verlag, New York, 1982, 87-102.

Dobson, A. 2004. Population Dynamics of Pathogens with Multiple Host-Species. Am. Nat. 164, S64-S78.

d'Onofrio, A. 2002. Stability properties of pulse vaccination strategy in SEIR epidemic model. Math. Biosci. 179, 57-72.

Dushoff, J., Plotkin, J. B., Levin, S. A., Earn, D. J. D. 2004. Dynamical resonance can account for seasonality of influenza dynamics. Proc. Natl. Acad. Sci. 101, 1691516916.

Earn, D. J. D., Rohani, P., Bolker, B. M., Grenfell, B. T. 2000. A Simple Model for Complex Dynamical Transitions in Epidemics. Science 287, 667-670.

Farkas, M., 1994 Periodic Motions. Springer-Verlag, New York.

Ferriere, R., Gatto, M. 1995. Lyapunov Exponents and the Mathematics of Invasion in Oscillatory or Chaotic Populations. Theor. Popul. Biol. 48, 126-171.

Greenman, J. V., Hudson, P. 1999. Host exclusion and coexistence in apparent and direct competition: An application of bifurcation theory. Theor. Popul. Biol. $56,48-64$.

Greenman, J. V., Kamo, M., Boots, M. 2004. External forcing of ecological and epidemiological systems: a resonance approach. Physica D 190, 136-151.

Greenman, J. V., Benton, T. G. 2005a. The frequency spectrum of structured discretetime population models; its properties and their ecological implications. Oikos 110, 369-389.

Greenman, J. V., Benton, T. G. 2005b. The Impact of Environmental Fluctuations on Structured Discrete Time Population Models: Resonance, Synchrony and Threshold Behavior. Theor. Popul. Biol. 68, 217-235. 
Grenfell, B. T., Bolker, B., Kleczkowski, A. 1995. Seasonality, demography and the dynamics of measles in developed countries. In: Epidemic models: their structure and relation to data. Mollison, D. ed. Cambridge: Cambridge University Press. 1995, 248-268.

Hale, J. K. 1969. Ordinary Differential Equations. Wiley-Interscience, New York. Hamilton, J. D. 1994. Time Series Analysis. Princeton University Press.

Heesterbeek, J. A. P., Roberts, M. G. 1995a. Threshold quantities for helminth infections. J. Math. Biol. 33, 415-434.

Heesterbeek, J. A. P., Roberts, M. G. 1995b. Threshold Quantities for Infectious Diseases in Periodic Environments. Journal of Biological Systems 3, 779-787.

Hethcote, H. W., Wang, W., Li, Y. 2005. Species Coexistence and Periodicity in Host-Host-Pathogen Models. J. Math. Biol. 51, 629-660.

Holt, R. D. 1977. Predation, Apparent Competition, and the Structure of Prey Communities. Theor. Popul. Biol. 12, 197-229.

Holt, R. D. 1984. Spatial Heterogeneity, Indirect Interactions and the Coexistence of Prey Species. Am. Nat. 124, 377-406.

Holt, R. D., Dobson, A. P., Begon, M., Bowers, R. G., Schauber, E. M. 2003. Parasite establishment in host communities. Ecology Letters 6, 837-842.

Ireland, J. M., Norman, R. A., Greenman, J. V. 2004. The effect of seasonal host birth rates on population dynamics: The importance of resonance. J. theor. Biol. 231, 229-238.

Kaitala, V., Ylikarjula, J., Ranta, E., Lundberg, P. 1997. Population dynamics and the colour of environmental noise. Proc. R. Soc. Lond. B264, 943-948.

Laakso, J., Kaitala, V. Ranta, E. 2004. Nonlinear biological responses to environmental noise affect population extinction risk. Oikos 104, 142-148.

Moneim, I. A., Greenhalgh, D. 2005a. Threshold and stability results for an SIRS epidemic model with a general periodic vaccination strategy. Journal of Biological Systems 13, 131-150.

Moneim, I. A., Greenhalgh, D. 2005b. Use of a periodic vaccination strategy to control the spread of epidemics with seasonally varying transmission rate. Mathematical Biosciences and Engineering 2, 591-611. 
Rinaldi, S., Muratori, S., Kuznetsov, Y. 1993. Multiple attractors, catastrophes and chaos in seasonally perturbed predator-prey communities. Bull. Math. Biol. 55, 15-35.

Roberts, M. G., Kao, R. R. 1998. The dynamics of an infectious disease in a population with birth pulses. Math. Biosc. 149, 23-36.

Ruel, J. J., Ayres, M. P. 1999. Jensen’s inequality predicts effects of environmental variation. Trends Ecol. Evol. 14, 361-366.

Shulgin, B., Stone, L., Agur, Z. 1998. Pulse vaccination strategy in the SIR epidemic model. Bull. Math. Biol. 60, 1123-1148.

Wichmann, M. C., Johst, K., Moloney, K. A., Wissel. C., Jeltsch, F. 2003. Extinction risk in periodically fluctuating environments. Ecological Modelling 167, 221-231.

Williams, B. G., Dye, C. 1997. Infectious disease persistence when transmission varies seasonally. Math. Biosc. 145, 77-88.

Wolfram, S. 1999. The Mathematica Book, Cambridge University Press.

\section{Appendix.}

\section{A. The rare invader approximation:}


Suppose the resident community (or web) is described by the population vector $\underline{\mathrm{x}}(\mathrm{t})$ and the species, community or web attempting to invade by $\mathrm{y}(\mathrm{t})$. Suppose further that the populations satisfy the dynamic equations:

$$
\mathrm{d} \underline{\mathrm{x}} / \mathrm{dt}=\underline{\mathrm{f}}(\underline{\mathrm{x}}, \underline{\mathrm{y}}, \mathrm{t}), \quad \mathrm{dy} / \mathrm{dt}=\mathrm{g}(\underline{\mathrm{x}}, \underline{\mathrm{y}}, \mathrm{t})
$$

If the invading population is sufficiently rare then we can approximate these equations by: $\quad \quad \underline{d x} / \mathrm{dt}=\underline{\mathrm{f}}(\underline{\mathrm{x}}, \underline{0}, \mathrm{t}), \quad \mathrm{dy} / \mathrm{dt}=\mathrm{G}(\underline{\mathrm{x}}, \underline{0}, \mathrm{t}) \cdot \underline{\underline{y}}$

where matrix $G$ is the first partial derivative of vector function $g$ with respect to $\mathrm{y}$, i.e $\mathrm{G}_{\mathrm{ij}}=\partial \mathrm{g}_{\mathrm{i}} / \partial \mathrm{y}_{\mathrm{j}}$. Matrix $\mathrm{G}$ is evaluated at $\mathrm{y}=\underline{0}$. The effect of external forcing on the invader can therefore be felt in two ways: Indirectly through the resident population $\underline{\mathrm{x}}$ and directly through its effect on the invader (as shown by the $t$ dependence in matrix G). The resident equations in (A2) are decoupled from the invader equations and can therefore be solved independently. The invader equations, which are linear in $\mathrm{y}$ but with time dependent parameters (i.e they are in general non-autonomous) can then be solved by substituting the solution for $\underline{x}$.

As an example, consider the predator-prey model:

$$
\begin{aligned}
& \mathrm{dx} / \mathrm{dt}=\mathrm{rx}(1-\mathrm{x} / \mathrm{K})-\mathrm{c}_{1} \mathrm{x} y \\
& \mathrm{dy} / \mathrm{dt}=\mathrm{c} \mathrm{x} \mathrm{y}-\mathrm{m} \mathrm{y}
\end{aligned}
$$

Here $\mathrm{x}$ denotes the prey and $\mathrm{y}$ the predator population. The biological meanings of the parameters in (A3) are explained in Table 1 (Model 1). The rare invader approximation yields:

$$
\mathrm{dx} / \mathrm{dt}=\mathrm{rx}(1-\mathrm{x} / \mathrm{K}), \quad \mathrm{dy} / \mathrm{dt}=(\mathrm{c} \mathrm{x}-\mathrm{m}) \mathrm{y}
$$


The (resident) prey population satisfies the logistic equation and the (invading) predator population satisfies a non-autonomous linear equation if the resident population $\mathrm{x}$ or one or more of the predator parameters is time varying.

\section{B. The condition for invasion.}

Define the exponent $\xi_{\text {ave }}$ by:

$$
\xi_{\text {ave }}=\lim ((\ln \|\mathrm{y}(\mathrm{t})\|) / \mathrm{t})
$$

where $\mathrm{y}(\mathrm{t})$ is the solution of (A2) for random initial vector and $\|\mathrm{y}(\mathrm{t})\|$ denotes the Euclidean length of vector $\mathrm{y}(\mathrm{t})$. The limit in (B1) is taken for large $\mathrm{t}$ (i.e. as $\mathrm{t}$ increases to infinity). Exponent $\xi_{\text {ave }}$ is the (leading) Lyapunov exponent and generalises the concept of leading eigenvalue for a non-autonomous linear system. (By leading eigenvalue we mean the eigenvalue with largest real part.) Invasion will take place if $\xi_{\text {ave }}>0$ but not otherwise. If $\mathrm{y}(\mathrm{t})$ is in fact scalar, i.e. the invader is unstructured, then equation (A2) for $\mathrm{y}(\mathrm{t})$ can be solved by integration and (B1) written as:

$$
\xi_{\text {ave }}=\lim \left(\frac{1}{t}\left(\ln \left(\frac{y(t)}{y(0)}\right)\right)=\lim \left(\frac{1}{t} \int_{0}^{t} G(\underline{x}(u), 0, u) d u\right)\right.
$$

If, in addition, $\mathrm{y}(\mathrm{t})$ is periodic and $\mathrm{G}$ in (A2) is linear in the resident populations (i.e. $\mathrm{G}=$ $\left.\mathrm{a}_{0}+\sum \mathrm{a}_{\mathrm{i}} \mathrm{x}_{\mathrm{i}}\right)$ then:

$$
\xi_{\text {ave }}=\mathrm{a}_{0 \mathrm{ave}}+\Sigma\left(\mathrm{a}_{\mathrm{i}} \mathrm{x}_{\mathrm{i}}\right)_{\mathrm{ave}}
$$

where the average is taken over a population cycle. For example, for Model 1 (Table 1), $\mathrm{a}_{0}=-\mathrm{m}$ and $\mathrm{a}_{1}=\mathrm{c}$.

We can rewrite the second term in (B2) using the identity: $\left(\mathrm{a}_{\mathrm{i}} \mathrm{x}_{\mathrm{i}}\right)_{\mathrm{ave}}=\left(\mathrm{a}_{\mathrm{i}}, \mathrm{x}_{\mathrm{i}}\right)_{\mathrm{cov}}+$ $\mathrm{a}_{\mathrm{iave}} \mathrm{X}_{\mathrm{iave}}$ where the first term is the covariance between predation parameter $\mathrm{a}_{\mathrm{i}}$ and 
resident population $\mathrm{x}_{\mathrm{i}}$. If $\mathrm{x}_{\mathrm{i} 0}$ denotes the ith resident (stable) population level when there is no forcing then (B3) can be rearranged as:

$$
\xi_{\text {ave }}=\xi_{0}+\sum \mathrm{a}_{\text {iave }}\left(\mathrm{x}_{\text {iave }}-\mathrm{x}_{\mathrm{i} 0}\right)+\Sigma\left(\mathrm{a}_{\mathrm{i}}, \mathrm{x}_{\mathrm{i}}\right)_{\mathrm{cov}}
$$

where $\xi_{0}=\mathrm{a}_{0 \mathrm{ave}}+\sum \mathrm{a}_{\mathrm{iave}} \mathrm{x}_{\mathrm{i} 0}$, namely the exponent when there is no forcing.

If the invader is structured than an explicit solution for invader $y(t)$ (in terms of average values) is in general not possible. Non-autonomous linear equations can only rarely be solved explicitly (Hale 1969). If forcing is periodic and the invader structured then one approach is to solve the equations for the Fundamental Matrix: $\Phi(t)$ (Farkas 1994):

$$
\mathrm{d} \Phi / \mathrm{dt}=\mathrm{G}(\underline{\mathrm{x}}(\mathrm{t}), \underline{0}, \mathrm{t}) \Phi(\mathrm{t})
$$

subject to the initial condition $\Phi(0)=$ I where I is the identity matrix. If $\mathrm{p}$ is the period of the population cycles then the eigenvalues (Floquet multipliers) of $\Phi(\mathrm{p})$ determine whether invasion takes place. The standard criterion applies: If the dominant eigenvalue of $\Phi(\mathrm{p})$ has magnitude greater than one then there will be invasion. In the text, matrix $\Phi(\mathrm{p})$ is labelled as Q.

\section{C: The storage effect:}

If in formula (3) we take $c=c_{0} E_{1}, N=N_{0} E_{2}$ where $E_{1}=E_{2}=(1+\delta \cos (2 \pi t / p))$ then:

$$
\xi_{\text {ave }}=\left(\mathrm{c}_{0} \mathrm{~N}_{0}\right) \frac{1}{\mathrm{p}} \int_{0}^{\mathrm{p}}\left(1+\alpha_{0} \cos (2 \pi \mathrm{t} / \mathrm{p})+\alpha_{1} \cos ^{2}(2 \pi \mathrm{t} / \mathrm{p})\right) \mathrm{dt}-\mathrm{m}_{0}
$$

where $\alpha_{0}=2 \delta, \alpha_{1}=\delta^{2}$. Integration in (C1) yields (4a). The integrand in (C1) is plotted in Fig 1a. If c, $N$ are out of phase, i.e. $E_{2}=(1+\delta \cos ((2 \pi t / p)+\pi))=(1-\delta \cos (2 \pi t / p))$ 
then $\alpha_{0}=0, \alpha_{1}=-\delta^{2}$. The integrand then has the graph shown in Fig 1b and (C1) gives (4b).

\section{The quadratic approximation.}

Suppose the invader equations can be written as: $d I / d t=$ M.I where $I=\left(I_{1}, I_{2}\right)^{T}, T$ denotes the transpose operation and:

$$
M=\left\lfloor\begin{array}{ll}
A & B \\
C & D
\end{array}\right\rfloor .
$$

The elements of $M$ are in general time dependent. Define $X=I_{1}, Y=I_{2} / I_{1}$ then:

$$
\mathrm{d}(\ln \mathrm{X}) / \mathrm{dt}=\mathrm{A}+\mathrm{B} \mathrm{Y} \quad(\mathrm{D} 1), \quad \mathrm{dY} / \mathrm{dt}=\mathrm{C}+(\mathrm{D}-\mathrm{A}) \mathrm{Y}-\mathrm{BY}^{2} \quad \text { (D2). }
$$

Equation (D2) for Y is uncoupled but nonlinear. Substitute in (D2) the expansion for Y in powers of the forcing strength $\delta$ :

$$
\mathrm{Y}=\mathrm{Y}_{0}+\delta \mathrm{Y}_{1}+\delta^{2} \mathrm{Y}_{2}+\ldots
$$

to generate a sequence of analytically solveable forced linear differential equations with constant coefficients for the components $\mathrm{Y}_{1}, \mathrm{Y}_{2}, \ldots$ Equation (D1) for $\mathrm{X}$ then yields an approximation for the leading Lyapunov exponent to any desired power of $\delta$. This solution can be generalised when square matrix $\mathrm{M}$ is of arbitrary size. 


\section{Figure Captions:}

Figure 1: Variation of the predator "birth rate" over a cycle when prey oscillations and forcing on predation are (a) in phase (b) out of phase. $\delta=1, \mathrm{c}_{0} \mathrm{~N}_{0}=1$. (See Appendix C and compare with results (4a), (4b).)

Figure 2: Model 5: areas of exclusion and coexistence. Vector label: (prey1, prey2, predator): 1 =persists, $0=$ excluded. For example, (101) means all populations persist except prey2.

Figure 3: Typical response curves for a system under periodic forcing. (a) Forcing strong enough to produce asymmetry in resonance peak at A. (b) Further increase in forcing strength leads to (i) overlap of peak A with itself, creating two attractors at $\mathrm{p} \sim$ 2.9 (Dotted line indicates region where a third dynamic state is unstable) (ii) emergence of subharmonic B with double the forcing period (2p), sufficiently distorted to create multiple states at $\mathrm{p} \sim 1.3$. (Graph labelling: 1p, 2p denote the period of the population cycle.)

Figure 4: Strong negative correlation between prey 1 response curve and downward prey 2 threshold shift (Model 5). Forcing (on growth rates) sufficiently strong to create multiple states for subharmonic at $\mathrm{p} \sim 11$. Threshold shift is scaled to facilitate comparison. 


\section{Table Captions:}

Table 1: Equations for models discussed in the main text. "expt" identifies the parameter that is varied in searching for the invasion threshold position.

Table 2: Threshold values of predation parameter $\mathrm{c}_{1}$ for predator prey Model 1. Entries (rows 2 to 5) are threshold values of parameter $c_{1}$ in Model 1 (Table 1) at the predator invasion threshold $\left(\xi_{\text {ave }}=0\right.$ ) when forcing has period $\mathrm{p}$, to be compared with the threshold value of $c_{1}$ (row 6 ) when unforced (i.e. at $\xi_{0}=0$ ). The threshold is lowered (raised) if the forced threshold value of $c_{1}$ is less (greater) than the unforced value. The first parameter set (Table 1, Model 1) applies to columns (scenarios) A, B, C, D; the second set to scenarios E, F when exponential forcing is used with $\delta_{2}=10.0$. (See (8) in text.) The last row refers to stochastic forcing and gives the threshold values of $c_{1}$ for white noise under scenarios A to D.

Table 3: Infection threshold values of transmission rate $\beta$ for SIRS Model 2. Entries are forced threshold values of $\beta$ for Model 2. Average parameter values given in Table 1, Model 2. Unforced and unvaccinated threshold at $\beta_{0}=1.25 . \mathrm{m}=$ (vaccination period/forcing period). Parameters $\gamma, \alpha$ kept fixed throughout. vacc $=$ vaccination. In column D, disease transmission is frequency dependent (cf with column B). In columns $\mathrm{E}, \mathrm{F}$ there is vaccination with $\delta_{2}=10.0$. To check similarity of behaviour, compare columns A, B, C (Table 3) with columns A, B, D (Table 2).

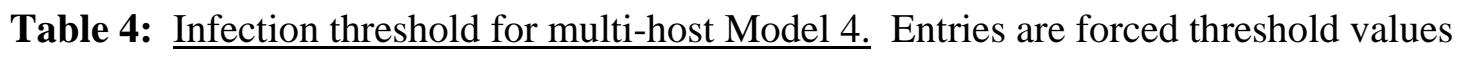
of $s_{1}=\beta_{21} / \beta_{11}$ for Model 4. Parameter values given in Table 1, Model 4. Columns C, $D$, E relate to control through host 1 only. 
Table 5: Invasion and Resonance in predator-prey-prey Model 5. Columns A, C: entries are forced threshold values of $c_{1}$; columns B, D: maximum values of prey 1 during a cycle. Parameter values given in Table 1, Model 5. 